\title{
Sprache als Schlüssel zum Bildungserfolg? Eine Fallstudie zur Reproduktion von Bildungsungleichheit im pädagogischen Alltag.
}

\section{Edina Krompàk}

Der vorliegende Beitrag fokussiert auf den Bildungsabschnitt im Leben eines mehrsprachigen Kindes, in dem der Übergang vom Kindergarten in die Primarschule ethnographisch untersucht wird. Am Beispiel von Dokumenten- und Praxisanalysen werden Selektionsprozesse und die Entstehung von Bildungsungleichheit auf der Mikroebene des pädagogischen Alltags insbesondere beim untersuchten Übergang rekonstruiert. Kontextuelle bildungspolitische Rahmenbedingungen werden dabei ebenfalls thematisiert.

\section{Einleitung}

Die Daten des vorliegenden Fallbeispiels wurden im Rahmen des Forschungsprojekts MEMOS (Mehrsprachigkeit und Mobilität im Übergang vom Kindergarten in die Primarschule in der Schweiz) der Pädagogischen Hochschule der Fachhochschule Nordwestschweiz (FHNW) erhoben und analysiert. Das Forschungsprojekt MEMOS entstand als Folgeprojekt des ethnographisch angelegten international vergleichenden Forschungsprojekts HELiE (Heterogenität und Literalität im Übergang vom Elementar- in den Primarbereich im europäischen Vergleich) der Universität zu Köln (Panagiotopoulou; 2010). Das Forschungsprojekt HELiE umfasst ethnographische Feldstudien, welche von 2007 bis 2009 in unterschiedlichen europäischen Bildungseinrichtungen (in der Vor- und Elementarschule) in Deutschland (Bundesland Rheinland-Pfalz) (Christmann \& Panagiotopoulou, 2012), Österreich (Graf, 2011), Finnland (Hortsch, 2010, 2011; Cuhls \& Panagiotopoulou, 2010) und Luxemburg (Christmann, 2009, 2011; Christmann \& Panagiotopoulou, 2012) durchgeführt wurden. Das HELiE-Projekt fragt «einerseits nach strukturellen Bedingungen und curricularen Voraussetzungen sprachlicher Bildung in den beteiligten Bildungssystemen und andererseits nach pädagogisch-didaktischen Alltagspraktiken zur Sprach(en)und Literalitätsförderung in spezifischen Kontexten von Bildungseinrichtungen, die sich in sozio-ökonomisch vergleichbaren Stadtteilen mit einem hohen Migra- 
tionsanteil befinden» (Cuhls \& Panagiotopoulou, 2010, S. 167). Im Mittelpunkt des HELiE-Projekts standen die folgenden Forschungsfragen:

- Wie wird in unterschiedlichen, separierend versus integrierend ausgerichteten sowie ein- und mehrsprachig organisierten europäischen Bildungssystemen beim Übergang in die Schule mit migrationsbedingter Heterogenität und Mehrsprachigkeit umgegangen?

- Wie ist sprachliche Bildung und (schrift-)sprachspezifische Förderung im Elementar- und Primarbereich unterschiedlicher Bildungssysteme curricular und strukturell konzipiert, wie wird sie im pädagogischen Alltag praktiziert und welche Bedeutung hat sie für das Lernen der (in der Regel mehrsprachig aufwachsenden) Kinder mit Migrationshintergrund (Hortsch \& Panagiotopoulou, 2010; Christmann \& Panagiotopoulou, 2012; Panagiotopoulou \& Krompàk, 2014, S. 51-52)?

Um einen internationalen Vergleich zwischen den ethnographisch erhobenen Daten zu ermöglichen, übernahm das MEMOS-Forschungsprojekt die methodologischen Grundlagen, das Forschungsdesign und die zentralen Forschungsfragen des HELiE-Projekts. Ausgehend von den Erkenntnissen des HELiE-Projekts und insbesondere von den Ergebnissen der in Luxemburg durchgeführten Studie (Christmann, 2011) erwies sich für das Schweizer Folgeprojekt die Untersuchung der Sprachförderung in den von Diglossie gekennzeichneten Bildungseinrichtungen (Panagiotopoulou \& Krompàk, 2014; Panagiotopoulou \& Kassis, in Druck) als zentrales Thema. Dementsprechend waren neben den oben aufgeführten Forschungsfragen die folgenden Fragen für das MEMOS-Forschungsprojekt von besonderer Bedeutung:

- Wie wird die bildungspolitisch und curricular geregelte Programmatik der sprachlichen Bildung insbesondere in Bezug auf die (Früh-)Förderung der Mehrsprachigkeit, der Standardsprache («Hochdeutsch») und des Dialekts («Schweizerdeutsch») im pädagogischen Alltag des Kindergartens und der Primarschule umgesetzt?

- Welche pädagogischen Praktiken im Umgang mit sprachlicher und soziokultureller Heterogenität lassen sich im Kindergarten und in der ersten Primarschulklasse beobachten? Mit welchen Qualitätsvorstellungen der Professionellen hängen diese Praktiken zusammen (Panagiotopoulou \& Krompàk, 2014, S. 53)?

Ähnlich wie im HELiE-Projekt wurden in der Schweiz Bildungseinrichtungen (Kindergarten und Primarschule) mit hohem Migrationsanteil ausgewählt. Von 2011 bis 2013 dauerten die intensiven Erhebungsphasen, die aus sechs Beobachtungsphasen von jeweils vier Wochen bestanden. Dabei begleiteten zwei Ethnographinnen beobachtend jeweils fünf Kinder mit verschiedenen Familiensprachen beim Übergang vom Kindergarten in die Primarschule (Panagiotopoulou \& Krompàk, 2014; Panagiotopoulou \& Kassis, in Druck). Neben den ethnographischen Protokollen und schriftlichen Dokumenten wurden Inter- 
views mit den Lehrpersonen, den Eltern und mit den Kindern in die Analyse und nachfolgende Interpretation einbezogen. Die Auswertung der Daten erfolgte nach der Forschungsstrategie der Grounded Theory (Charmaz 2006; Strauss \& Corbin 1996), deren methodologische Prämisse der Offenheit sowohl die Beschreibung als auch die Rekonstruktion des untersuchten pädagogischen Alltags ermöglicht.

Der für diesen Beitrag ausgewählte Einzelfall steht einerseits im engen Zusammenhang mit dem Gesamtprojekt, da in der Fallanalyse die Fragestellungen der beiden Projekte in Bezug auf die migrationsbedingte Heterogenität aufgegriffen werden. Andererseits werden im Einzelfall neue, im Gesamtprojekt kaum untersuchte Aspekte der Selektion erläutert. Der Beitrag erlaubt durch die Analyse des Bildungsweges eines Kindes Selektionsprozesse beim Übergang vom Kindergarten in die Primarschule zu rekonstruieren und Einsichten in die Reproduktion von Bildungsungleichheit im pädagogischen Alltag zu erhalten. Der vorliegende Beitrag geht der Frage nach, wie Bildungsungleichheit in der pädagogischen Praxis reproduziert und wie diese von den Akteurinnen und Akteuren begründet wird sowie welche Rolle die Deutschkenntnisse der Kinder mit Migrationshintergrund bei der Selektion spielen.

Im Folgenden werden zunächst methodologische Überlegungen zum Fallbeispiel erörtert und für den Forschungszusammenhang relevante wissenschaftliche Diskurse zu Selektion und Bildungsungleichheit aufgeführt. Anschliessend werden Analysen von Dokumenten, Beobachtungssequenzen und Interviewausschnitte vor dem Hintergrund theoretischer Bezugspunkte und curricularer Rahmenbedingungen der Bildungsinstitutionen beleuchtet. Der Beitrag schliesst mit einem Resümee ab, welches die Erkenntnisse des Fallbeispiels in Bezug auf die Fragestellung zusammenfasst. ${ }^{1}$

\section{Bildungsungleichheit aus ethnographischer Perspektive - Methodologische überlegungen}

Neben den internationalen PISA-Studien, die auf die soziale Selektivität des Bildungssystems aufmerksam machten (Baumert \& Schümer, 2001; Stanat, 2003; OECD, 2006), untersuchten Studien im deutschsprachigen Raum die Bedeutung des Übertritts in die nächsthöhere Bildungsinstitution für die Entstehung der Bildungsungleichheit (Gomolla \& Radtke, 2002; Kronig, Haeberlin \& Eckhart, 2000; Kronig, 2003, 2007). Insbesondere Kinder und Jugendliche mit Migrationshintergrund sind von den Mechanismen direkter und indirekter institutioneller Diskriminierung betroffen (Gomolla \& Radtke, 2002) und werden dementsprechend überdurchschnittlich oft in Sonderklassen und niedrigere Schultypen überwiesen (Kronig et al, 2000; Kronig, 2003, 2007). Die empirischen Resultate der oben erwähnten, vor allem quantitativ durchgeführten Studien erklären die Disparitäten auf der Makroebene. Welche Erkennt- 
nisse kann die ethnographische Forschungsstrategie in der Untersuchung der Ungleichheitsgenese bringen? Die ethnographische Forschung als «synchrone Beobachtung lokaler Praxis» (Hirschauer \& Amman1997, S. 23) ermöglicht die Untersuchung pädagogischer Praktiken auf der Mikroebene und eröffnet damit neue Einsichten, hat allerdings auch methodologische Grenzen. Im Beitrag von Diehm et al. werden diese Grenzen der Abbildung von Ungleichheit diskutiert (Diehm, Kuhn, Machold \& Mai, 2013). Um gesellschaftliche Ungleichheitsverhältnisse zu erklären, reicht die Analyse situierter Praktiken und Interaktionen nicht aus. Diesem methodologischen Kritikpunkt kann mit «mikroanalytischen in situ-Beobachtungen durch ein längsschnittliches ethnographisches Untersuchungsdesign» (ebd. S. 646) entgegengewirkt werden. Ähnlich wie in der Studie von Diehm, Kuhn, Machold und Mai (2013) wurde hier sowohl ein längsschnittliches ethnographisches Design eingesetzt als auch der systematische Einbezug von Artefakten in die Analyse gewährleistet. Um «die institutionelle Ordnung in Gestalt der translokalen Verflechtungen lokaler Ereignisse» zu rekonstruieren (Neumann, 2013, S. 19) wurden die Ansätze der institutional ethnography (Smith, 2005; Nadai \& Koch, 2011) in der Analyse berücksichtigt. Die institutional ethnography verortet einerseits die Handlungen der Akteurin, des Akteurs in institutionellen Kontexten, andererseits erzielt Erkenntnisse über die Institution zu gewinnen (Smith, 2005). Aus diesem Grund werden bei der Interpretation der Daten die kontextuellen bildungspolitischen Rahmenbedingungen mitberücksichtigt. Um die Bildungskarriere von Dilek ${ }^{2}$ zu rekonstruieren, werden die empirischen Daten (Analyse von Artefakten und in situ-Beobachtungen) im Folgenden chronologisch aufgeführt.

\section{Bildungskarriere von Dilek aus ethnographi- scher Perspektive 3}

\section{Schuleignungstest als Instrument für die Selektion} Der Horgener Schuleignungstest (Krebs, 1984), der im untersuchten Kindergarten zentral angeordnet eingesetzt wird, erfasst den kognitiven Entwicklungsstand anhand von Form- und Begriffsbildung, seriellem und logischem Denken sowie Graphomotorik, Arbeitshaltung und Instruktionsverständnis bei 5- bis 6-jährigen Kindern (Annen, Brunner, Sollberger, 2005) und hat somit das Ziel, deren Schuleignung zu prüfen. Diejenigen Kinder, die den Schuleignungstest nicht bestehen, werden in die sogenannte Einführungsklasse eingeteilt. Die Einführungsklasse, die nicht in allen Deutschschweizer Kantonen angeboten wird, setzt sich zum Ziel, Schülerinnen und Schülern mit Lernschwierigkeiten einen langsamen Einstieg in die Schule zu ermöglichen:

Die Einführungsklasse fördert Schülerinnen und Schüler, deren Schulfähigkeit noch nicht in allen Bereichen entwickelt ist. Der Schulstoff des ersten Schuljahres wird auf zwei Jahre verteilt. Diese zählen als ein Schuljahr. Am 
Ende des zweiten Schuljahres erfolgt der Übertritt in die 2. Regelklasse, in die integrative Schulungsform (ISF) oder in die Kleinklasse. ${ }^{4}$

Dilek, ein fünfjähriges Mädchen mit der Familiensprache Türkisch, geriet während der Feldphase im Kindergarten wegen ihrer Mehrsprachigkeit und der resultierenden besonderen Aufmerksamkeit der Lehrperson in ihrer Entwicklung in den Mittelpunkt meines Interesses. Diese Aufmerksamkeit galt vor allem für den Übergang vom Kindergarten in die Primarschule: Die Frage lautet, ob Dilek der Eintritt in die erste Primarschule gelingen würde. Darüber sollte der Horgener Schulleistungstest (Krebs, 1984) entscheiden, der im Januar 2012 (ein halbes Jahr vor dem Übertritt) im Kindergarten durchgeführt wurde. Dilek bestand den Schuleignungstest nicht.

Auf meinen Wunsch hin habe ich von der Lehrperson die Tests der Kinder erhalten und bekam so einen Einblick in Dileks Leistungen. Da der Schuleignungstest an einem unangekündigten Tag stattfand, an dem ich nicht im Kindergarten war, konnte ich keine Feldnotizen zur Durchführung des Tests machen. Bei der Analyse des Tests (post situ) wählte ich eine Aufgabe aus, die mich einerseits wegen ihrer Aufgabenstellung andererseits wegen der Ausführung von Dilek irritiert hat. Die Aufgabenstellung des Schuleignungstests bestand darin, dass die Kinder in sechs bis sieben Minuten mit Bleistift einen «schönen grossen Mann» (Krebs, 1984, S. 6) zeichnen sollten. ${ }^{5}$ Der Zeitraum (sechs bis sieben Minuten), die Farbauswahl (keine Farbe) und das Thema (ein schöner grosser Mann) waren im Schuleignungstest klar vorgegeben. Bei der Bewertung der Aufgaben zählten die folgenden Punkte (Krebs 1984, S. 10-11): Beine in richtiger Zahl vorhanden (1), Arme in richtiger Zahl vorhanden (2), Rumpf vorhanden (3), beide Arme und Beine mit Rumpf verknüpft (4), beide Arme und Beine zweidimensional, länger als breit (5), Füsse vorhanden (6), Kleidung vorhanden (7), Finger vorhanden (8), richtige Anzahl Finger (9), Hände vorhanden (10). Nach den Kriterien des Schuleignungstests zeigen sich bei Dilek bei den Punkten 5, 7, 9 und 10 Mängel (Abbildung 1). Aus der Gesamtpunktzahl zehn erreichte sie bei dieser Aufgabe sechs Punkte.

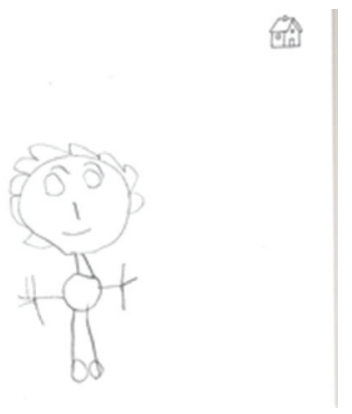

Abbildung 1 Zeichnung von Dilek im Schuleignungstest, Januar 2012 
Während meiner Feldphase im Kindergarten ist mir aufgefallen, wie kreativ Dilek zeichnet und wie oft sie wegen dieser Fähigkeit von der Lehrperson gelobt wurde. Deshalb war die Zeichnung von Dilek im Schuleignungstest eine Überraschung, aber auch gleichzeitig eine Irritation für mich. Einen Monat nach der Durchführung des Schuleignungstests konnte ich eine Szene beobachten, in der Dilek einen Waggis, eine traditionelle Basler Fastnachtsfigur, gezeichnet hat:

Vor dem Morgenkreis sitzen die Kinder an verschiedenen Tischen und beschäftigen sich mit Zeichnen oder mit Puzzles. Dilek sitzt an einem Tisch mit Luana und Sahanti. Ich sitze Dilek gegenüber und beobachte, was sie malt. Luana sitzt rechts von mir und versucht, ein Holzpuzzle zusammenzulegen. Sahanti und Dilek zeichnen. Dilek zeichnet eine Figur mit rosarotem Kopf, grossen Augen, lila Haaren und einer grossen roten Nase. Die Lehrperson kommt vorbei, schaut auf die Zeichnung und lobt Dilek «Schön, Dilek!» Dann wendet sie sich mir zu und sagt: «Das kann sie gut. Alles was visuell ist, kann sie gut, aber mit dem Auditiven hat sie Probleme.» Dilek zeichnet weiter und ich frage sie, wer diese Figur auf ihrer Zeichnung sei. Sie sagt, dass es ein Waggis sei. Die Waggis sind lustig-böse Figuren in der Basler Fasnacht. Das Thema Fasnacht wurde im Kindergarten in den letzten Tagen oft behandelt und die Kinder haben viele Fotos über Waggis gesehen und nachgezeichnet. Die Kleider des Waggis zeichnet Dilek sehr bunt, sogar die Streifen an den Socken haben verschiedene Farben. Die Anzahl der Finger stimmt an beiden Händen. Ich achte besonders darauf, da Dilek im Schuleignungstest die Anzahl Finger nicht richtig markiert hat. Dann zeichnet sie orange, rosarote und blaue Flocken auf der rechten Seite des Waggis. Ich frage sie, was das sei. Sie antwortet: «Konfettis». (Protokoll vom 13. Februar 2012, EK.)

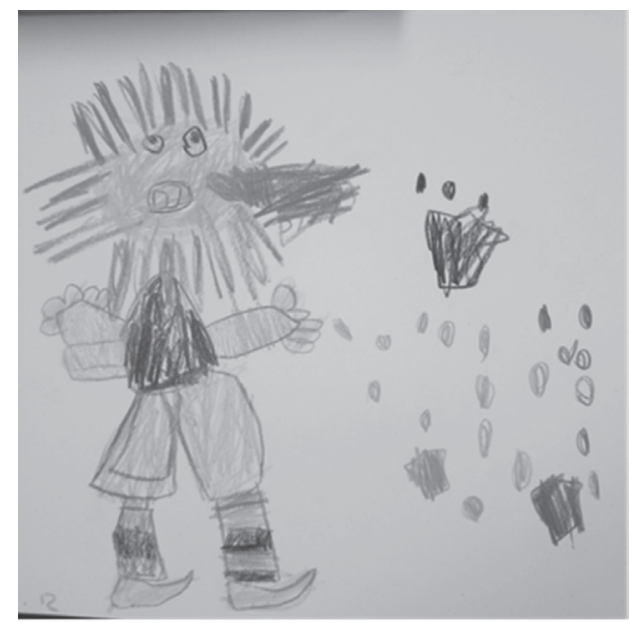

Abbildung 2: Waggis - Eine Zeichnung von Dilek (13. Februar 2012) 
Das Bild von Dilek zeichnet sich durch gelungene Farbkomposition und verhältnismässige Körperproportionen aus. Im Gegensatz zur gezeichneten Figur im Schuleignungstest stellt Dilek den Waggis mit zweidimensionalen Beinen und Armen, mit Verkleidung und mit der richtigen Anzahl Finger dar. Die Analyse der Zeichnung von Dilek zeigt, dass der Horgener Schuleignungstest (Krebs, 1984) mit seiner Aufgabenstellung und dem Kriterienraster nicht den aktuellen Entwicklungsstand der Kinder erfasst. Demzufolge kann ein Kind, das die obige Aufgabe «einen schönen grossen Mann» zu zeichnen (Krebs, 1984, S. 6) nicht erfolgreich löst, durchaus in der Lage sein, in einer anderen Situation eine Zeichnung zu erstellen, die alle Kriterien dieser Aufgabe des Schuleignungstests erfüllt. Darüber hinaus ist der Schulleistungstest monolingual ausgerichtet und berücksichtigt die Familiensprachen der untersuchten Kinder nicht (Diehm, Kuhn, Machold \& Mai, 2013).

Im Weiteren ist es von Bedeutung, die Einstellung der Lehrperson gegenüber dem Schuleignungstest kennenzulernen und zu analysieren, welchen Stellenwert dieser in ihrer pädagogischen Praxis hat:

Ja, also der Schulreifetest ist eigentlich nur etwas, das man den Ki- den Eltern etwas hinlegen kann und zeigen kann: Das ist nicht so gut. Ich weiss jetzt nicht mehr auswendig, wie viel Punkte sie gemacht haben, auf jeden Fall, es reichte nicht. Von den Punktezahlen her reichte es nicht. Aber ich selber brauche eigentlich keinen Schulreifetest, um den Eltern zu sagen, hier in diesem Bereich und das und das und das geht und das geht nicht. Weil, wir haben die Kinder zwei Jahre lang. Und dann geht das auch ohne Schulreifetest. Würde es gehen. Aber es ist für uns einfach noch wichtig, weil wir sagen können: Schauen Sie da ((tippt auf den Tisch)). Im Vergleich zu diesem Kind, das gleich alt ist. Oder ein Kind das jetzt wie Hasanthi noch bei den Kleinen ist und viel viel mehr Punkte gemacht hat im ersten Jahr als die zwei, die in die Schule kommen sollten. Das ist zum Vergleichen schon noch- für die Eltern erfassbar (Interview mit der Lehrperson, Kindergarten, 4. Juni 2012, EK).

Aus der Argumentation der Lehrperson geht hervor, dass der Schuleignungstest in ihrer pädagogischen Praxis ein Legitimationsinstrument darstellt. Mit dem Schuleignungstest wird die Selektionsentscheidung der Lehrperson gegenüber den Eltern begründet. Im Weiteren stützt die Lehrperson ihre Entscheidung mit bezugsnorm-orientierter Argumentation, die wiederum die individuelle Entwicklung des einzelnen Kindes ausser Acht lässt. Ein wichtiges Kriterium stellt dabei das Alter dar, anhand dessen die Leistungen der Kinder verglichen werden. Dabei greift die Lehrperson auf das reifungstheoretisch-nativistische Erklärungsmuster zurück, welches dem Kind eine passive Rolle zuweist und die Reifung und das Alter des Kindes als zentrale Kriterien bei der Einschulung berücksichtigt (Stamm, 2013). Stamm (2013) weist auf die Unzuverlässigkeit der Schulreifetests hin und zitiert eine Meta-Analyse von La Paro und Pianta 
(2001), deren Ergebnisse über die erfolgreiche Bewältigung des ersten Schuljahres bei vielen nicht schulreif diagnostizierten Kindern berichten.

Nach Stamm (2013) berücksichtigen die Lehrpersonen beim Übergang vom Kindergarten in die Primarschule zunehmend die sprachlichen Vorläuferfähigkeiten der Kinder (Mauron Schläppi, 2009; Maryland School Readiness Report, 2011, zitiert nach Stamm, 2013). In diesem Sinne scheint es relevant zu sein, wie die Lehrperson die sprachliche Fähigkeit von Dilek bzw. deren Bedeutung für den Schulübertritt einschätzt:

Sprachlich sind sie gar nicht schlecht. Die reden halt von Anfang an nur Hochdeutsch. [...] Da liegt das an anderen Sachen, weil die haben als kleine Kinder einfach zu wenig zu Hause mithelfen dürfen und die haben viele Handlungsabläufe erst mal lernen müssen. Also die haben eine rechte Entwicklung durchgemacht, wenn ich denke, wie sie am Anfang waren, da ging gar nichts. Und jetzt geht doch, es geht wirklich einiges und es ist einiges gegangen, aber von der Sprache her, sprechen tun sie nicht schlecht, aber ich denke das Mädchen [Dilek] hat einfach Probleme, das Gehörte umzusetzen. Und zwar nicht das Deutsche. Sondern einfach, was sie akustisch hört, geht vom Hirn aus nicht in die richtige Richtung. Dann versteht sie es nicht. Wenn sie es hört und sieht, dann kann sie es. Aber wenn ich nur verbal an alle Kinder einen Auftrag gebe, dann kann sie es nie [...] Sozial sind sie gut. Also gut, sie sind ganz brave Kinder. Über-brav. Wollen alles immer recht machen, streiten kaum, also Wunschkinder. Also wenn man solche Kinder in der Schule hat, die sind ja toll (Interview mit der Lehrperson, Kindergarten, 4. Juni 2012, EK.).

Im Interview betont die Lehrperson, dass in die Einführungsklasse empfohlene Kinder keine schlechten sprachlichen Fähigkeiten auswiesen. Der Grund, weshalb diese Kinder, insbesondere Dilek, in die Einführungsklasse eingeteilt werden sollen, liegt an anderen Aspekten. In diesem Fall äussert sie ihre subjektive Überzeugung zum (Nicht-) Vorhandensein des Mithelfens zu Hause. Dadurch, dass Dilek vor allem durch ihre Schwächen wahrgenommen wird (Schwierigkeiten im akustischen Bereich), spielen ihre Stärken, wie gute mündliche Sprachfähigkeit und erfolgreiche Umsetzung visueller Lernaufträge sowie ihre hohen sozialen Kompetenzen bei der Einteilung in die Einführungsklasse keine relevante Rolle (Protokoll vom 13. Februar 2012 und Interview vom 4. Juni 2012). Parallel dazu wird der rasante Lernzuwachs von Dilek nicht in die Prognose einbezogen. Im Fall von Dilek stellt nicht die mangelhafte Beherrschung des Deutschen den Grund für die Empfehlung für die Einführungsklasse dar.

\section{Reproduktion von Bildungsungleichheit}

Während meiner Feldphase im Kindergarten (Oktober 2011 bis Juni 2012) wurde ich in regelmässigen Abständen in in-situ-Gesprächen mit den Lehrpersonen über den gegenwärtigen Stand von Dileks Fall in Bezug auf die Einführungsklasse informiert. Es gab mehrere Gespräche zwischen den Lehrpersonen 
und dem Vater sowie der Schulleitung der zukünftigen Primarschule. In diesen Gesprächen ging es darum, den Vater zu überzeugen, Dileks Eintritt in die Einführungsklasse zuzustimmen.

Die Einführungsklasse erscheint im Bildungsgesetz des Kantons BaselLandschaft ${ }^{6}$ ( $\$ 45$, Abs. 3) unter dem Begriff spezielle Förderung. Nach dem Bildungsgesetz setzt die spezielle Förderung «eine vorherige Abklärung durch eine vom Kanton bestimmte Fachstelle voraus» 7 . Diese Abklärung hat jedoch im Einverständnis mit den Erziehungsberechtigten zu erfolgen. Wiederum entscheidet «über die Aufnahme einer Speziellen Förderung [...] die Schulleitung im Einverständnis mit den Erziehungsberechtigten. In die Einführungsklasse ist eine Aufnahme gemäss $\$ 25$ Absatz 3 auch ohne Einverständnis der Erziehungsberechtigten möglich» ${ }^{8}$. Falls die Eltern mit der speziellen Förderung nicht einverstanden sind und sich weigern, eine Abklärung durchzuführen, können die Kinder nicht ohne Einverständnis in die Einführungsklasse eingeteilt werden. Liegt aber eine Abklärung vor, welche die spezielle Förderung begründet, kann die Schulleitung den Besuch der Einführungsklasse ohne das Einverständnis der Eltern anordnen.

Im Fall von Dilek lehnte der Vater sowohl die Einführungsklasse als auch die Abklärung ab. Kurz vor dem Übertritt in die erste Klasse am 5. Juli 2012 konnte ich Dileks Vater zu seinem familiären Hintergrund und seiner Einstellung bezüglich der Einführungsklasse interviewen:

V: Also, mein Vater ist vor ungefähr 35 Jahren in die Schweiz gekommen. //Ja.// Ja, also eigentlich bin ich zweite Generation, aber ich bin schon hier geboren in der Schweiz, also geboren, alles. Die Schule habe ich alle hier besucht. Kindergarten und alles [...]

I: Haben Sie Erfolgserlebnisse in der Schule gehabt? Und Schwierigkeiten? V: Also die Schwierigkeiten - ich fang mal mit den Schwierigkeiten an - die habe ich gehabt vom eh, vom Kindergarten bis in die zweite Klasse. Das waren eigentlich nur hauptsächlich sprachliche Probleme. Da habe ich viel Deutschkurs besucht und so. Ich ging auch in die kleine Klasse. Die Einfuihrungsklasse war das, die EK, fürs erste. Und nachher hab ich eh, viele Kurse besucht und hab auch viel Unterstützung bekommen und dann ging ich dann in die, die normale grosse Klasse in die Zweite und dann eh ging's nur noch positiv bergauf (Interview mit dem Vater von Dilek, 5. Juli 2012, EK).

Der Vater von Dilek gehört zur zweiten Generation von Migrantinnen und Migranten, die in der Schweiz geboren und aufgewachsen sind. Im Interview berichtet er über seine anfänglichen sprachlichen Schwierigkeiten im Kindergarten und in der ersten Primarschulklasse. Aus eigener Erfahrung kennt er die Einführungsklasse (EK) oder in der damaligen Bezeichnung «die kleine Klasse». Er kategorisiert die Klassen als «normale» und «Einführungsklasse». Als Kind von Eltern mit Migrationshintergrund besuchte er mehrere Deutschkurse und erhielt Deutschförderung in der Schule. Eine bedeutende positive Veränderung in der Sprachentwicklung erfuhr der Vater von Dilek erst in der zweiten Klasse, 
in der es nur noch "positiv bergauf» (siehe oben) ging. Der Grund, weshalb er seine Kinder trotzdem nicht in die Einführungsklasse einteilen liess, kristallisierte sich aus einer späteren Interviewsequenz heraus:

I:Und wie finden Sie die Einführungsklasse? Sie haben gesagt, Sie haben das selber erlebt. V: Jawohl. Eh, ehrlich- Also ich hab ja das selber erlebt. ((Räuspert sich.)) Da habe ich nicht so positive Erlebnisse gehabt in der Einführungsklasse. I: Warum? V: Weil eh, wo ich in der Einführungsklasse war, da wurden wir gehänselt von den Kindern. Weil wir eine kleine Gruppe waren. Wir waren immer die Aussenseiter und- Um- Als Kind, wenn man das so spürte eh, tut schon weh. //Mhm.// Ja, und Kinder können manchmal gemein se- Also gemein sein, ja, das ist es- Weil eh, man ist in einer kleinen Gruppe und wenn man da ausgeschlossen ist von den anderen, zum Beispiel so Spieltage oder in den Pausen und so. Oder wenn man mit anderen Kindern spielen will, privat und dann sagt, sagt der eine: Ja, die geht da in die kleine Klasse, mit dem musst du nicht spielen. Die sind anders und so. Ja (Interview mit dem Vater von Dilek, 5. Juli 2012, EK).

Da der Vater von Dilek selber in der Einführungsklasse war, erlebte er eine Ausgrenzung in der Schule, die sich auch auf die ausserschulischen Kontexte übertragen hat. Mit der Einführungsklasse wurden soziale Differenzen hergestellt, zwischen den "guten» und «schlechten» Schülerinnen und Schülern. Nach West und Fenstermaker (1995) äussert sich doing difference in andauernden interaktionalen Ausführungen («ongoing interactional accomplishment») (ebd. S. 9). Die Kategorien Gender, Herkunft und Klasse sind bedeutende Elemente unseres sozialen Lebens und können als Teil der Identität in Abhängigkeit vom Kontext stärker oder schwächer betont werden (ebd. S. 33). Es besteht nicht nur eine Herstellung von Differenzen innerhalb der Schulklassen, sondern auch die Konstruktion des Anderen, das othering (Jäger, 2011). Diejenigen Kinder, die die Einführungsklasse besuchen, sind nicht nur anders, sondern «schlechter», «mit dem musst du nicht spielen» (Interview mit dem Vater von Dilek, 5. Juli 2012, EK). Beachtet man, dass vor allem Kinder mit Migrationshintergrund die Einführungsklasse besuchen, werden soziale Differenzen nicht nur hergestellt, sondern soziale Ungleichheit reproduziert (Bourdieu \& Passeron, 1971). Der Vater von Dilek möchte seiner Tochter das Trauma der Einführungsklasse, welches er selbst erlebt hat, ersparen. Die eigenen Ausgrenzungserfahrungen lenken und strukturieren dabei sein Handeln.

\section{Sprache als Schlüssel zum Bildungserfolg?}

Im Januar 2013, ein halbes Jahr nach dem Übertritt, habe ich in einem in-situ-Gespräch von Dileks Lehrperson (der ersten Primarschulklasse) erfahren, dass sie doch in die Einführungsklasse eingeteilt werden soll:

Vor dem Unterricht, kurz vor 8 Uhr komme ich ins Klassenzimmer, begrüsse den Lehrer und frage, wie es ihm geht. Er beginnt über die Elterngespräche 
zu erzählen. Er hat schon zehn Elterngespräche durchgeführt, jetzt hat er nur noch wenige. $\mathrm{Zu}$ meiner Frage, ob die Eltern zufrieden seien, antwortet er, dass die meisten Eltern zufrieden seien. Er hat versucht, dem Vater von Dilek klar zu machen, dass Dilek besser in der Einführungsklasse aufgehoben wäre. In der Einführungsklasse ist das Tempo langsamer und das wäre für Dilek besser. Sie sei zu langsam und kann nicht Anweisungen durchführen. Sie sei ständig auf die Hilfe von anderen Kindern angewiesen. Der Vater von Dilek weigert sich und sagt, dass Dilek ihm nie verzeihen würde, wenn er sie aus der Klasse herausnehmen würde. Die Lehrperson beteuert, dass sie so nichts machen kann, wenn sich die Eltern wehren. (Protokoll des in-situ-Gesprächs, 15. Januar 2013, EK).

Die Begründung der Lehrperson für die Empfehlung in die Einführungsklasse liegt in der langsamen Ausführung der Aufgaben und im mangelnden Instruktionsverständnis von Dilek. Ausserdem sei sie beim Lösen von Aufgaben auf andere Kinder angewiesen. Hier äussert sich ein Bildungsverständnis, das nicht die Ko-Konstruktion des Lernens im Vordergrund hat, sondern ein bestimmtes Lerntempo und das Lösen einer Aufgabe ohne Hilfe als Anforderung formuliert.

Obwohl Dilek auch von der Lehrperson für Deutsch als Zweitsprache als äusserst motivierte Schülerin wahrgenommen wird, widerspiegelt sich diese Wahrnehmung nicht in deren Beurteilung: "Dilek ist sehr sehr motiviert. Sie streckt auch sehr oft auf. Sie ähm ist mitteilungsbedürftig ähm (5) auch wenn sie es nicht weiss. //I: Mhm// Sie hat auch keine Angst vor Fehlern. //I: Mhm/l Das kam erst mit der Zeit (...), hab ich das Gefühl» (Interview mit der DaZ-Lehrperson, 27. Juli 2013, EK).

Trotz der hohen Motivation erreicht Dilek die Lernziele der ersten Klasse «nicht wirklich», wie sich die Klassenlehrperson äussert: "Also, dass sie [Dilek und Erkan] (7) einfach erstens mal eben Deutsch und Sprache, wo sie halt sebr an der Grenze sind oder eigentlich eben ein bisschen darunter also halt einfach von den Lernzielen, die sie halt einfach nicht wirklich erreichen. Und ich denke, da wäre halt wirklich eine Einführung, also EK [Einführungsklasse], wo sie die zwei Jahre einfach die erste Klasse gemacht hätten, wo sie einfach länger Zeit gehabt hätten, weil ich habe das Gefühl, sie sind sehr sehr kindlich»9 (Interview mit der Klassenlehrperson, 11. Juni 2013, EK).

Im Interview mit der Klassenlehrperson werden die Leistungen von Dilek in der Sprache in Bezug auf die Lernziele beschrieben. Es fällt auf, dass innerhalb eines Satzes die Nähe der Leistungen zu den Lernzielen mit wachsendem Abstand beschrieben werden: "an der Grenze», "bisschen darunter», "nicht wirklich erreicht» (Interview mit der Klassenlehrperson, 11. Juni 2013, EK). Im Weiteren argumentiert die Lehrperson mit der «Reife» von Dilek. Neben der "Reife» und den erreichten Lernzielen kommt noch ein weiterer Aspekt für den erfolgreichen Übertritt in die zweite Klasse hinzu, welchen die DaZ-Lehrperson im Interview formuliert:»[...]und [ich] kann sie nicht jeden Tag begleiten und ähm grad was die Arbeitshaltung betrifft, sagen welches Blatt, welches Mäppli, wo 
das hingehört, das sind so Grundvoraussetzungen, wo die sie (4) als Übergang in die zweite Klasse eigentlich beherrschen müssten //I: Mhm// und das ist leider nicht vorhanden" (Interview mit der DaZ-Lehrperson, 27. Juli 2013, EK).

Unter dem Begriff "Arbeitshaltung" wird die Organisationskompetenz von schulischen Artefakten (Arbeitsblätter und Mappen) verstanden, die zum Beispiel hilft, mit den vielen Arbeitsblättern zurechtzukommen. Ähnlich dem Instruktionsverständnis im Kindergarten kommt der Organisationskompetenz von schulischen Artefakten in der Schule eine besondere Bedeutung zu und scheint noch vor den kognitiven Fähigkeiten als Grundvoraussetzung den ersten Platz für den Übertritt in die zweite Klasse einzunehmen. Dadurch erscheint die Schule vor allem als Sozialisationsinstanz und weniger als Bildungsinstitution im engeren Sinne des Wortes.

Am Ende des Schuljahres erhielt Dilek keinen Stundenplan für die zweite Klasse, da sie die erste Klasse wiederholen muss. Nach der Beurteilung der Klassenlehrperson und der Lehrperson für Deutsch als Zweitsprache sollte sie in die Einführungsklasse gehen. Da der Vater von Dilek die Einführungsklasse wieder abgelehnt hatte, blieb für sie jedoch nur die Möglichkeit, die erste Klasse zu wiederholen.

\section{Resümee}

Das Fallbeispiel von Dilek ermöglicht eine vertiefte Analyse der pädagogischen Praxis beim Übertritt vom Kindergarten in die Primarschule aus ethnographischer Perspektive. Obwohl Dilek die curricularen Bedingungen der sprachlichen Bildung im Kindergarten nach der subjektiven Einschätzung der Lehrperson erfüllt, scheitert sie am Schuleignungstest (Krebs, 1984). Ausgewählte Teilbereiche des Tests wurden analysiert und mit Dokumenten (Zeichnungen) von Dilek verglichen. Der Schuleignungstest zeigte im untersuchten Bereich nicht den aktuellen Entwicklungsstand des Kindes. Darüber hinaus wirft der Schuleignungstest aus dem Jahr 1984 (Krebs, 1984) Fragen für den geeigneten Einsatz in sprachlich und kulturell heterogenen Kindergartengruppen auf (Diehm, Kuhn, Machold \& Mai 2013). Die beim Schulerfolg als relevant erachtete gut ausgebildete Sprachkompetenz verliert, sofern vorhanden, an Bedeutung bei der Selektionsentscheidung. In diesem Fall gewinnen andere Faktoren, die die Sozialisationsaufgabe der Schule unterstützen, an Bedeutung und werden zentral beim Übergang vom Kindergarten in die Primarschule.

Im Kindergarten wird der Schuleignungstest von den Kindergartenlehrpersonen als Legitimations-und Selektionsinstrument eingesetzt, um die Überweisung in die Einführungsklasse vor den Eltern zu legitimieren. Dadurch tritt der Kindergarten als erste Selektionsinstanz im Bildungssystem auf, die Kinder nach bestimmten Kriterien der Regelklasse oder der Einführungsklasse zuordnet. Nicht die globale Sprachkompetenz, sondern insbesondere die «Schulreife» und 
das Instruktionsverständnis entscheiden darüber, ob jemand in die erste Klasse kommen kann. Hier muss hervorgehoben werden, dass der in der pädagogischen Praxis verwendete Begriff der «Schulreife» nicht dem aktuellen wissenschaftlichen Diskurs entspricht. Die Einschulung und auch die Einteilung in die Einführungsklasse erfolgt dementsprechend auf der Basis der reifungstheoretischen Sichtweise (Stamm, 2013), die als überholt gilt (Stamm, 2013; Barth, 2012). Das Kriterium Schulfähigkeit ist nach aktuellen Erkenntnissen «multidimensional» (Stamm, 2013, S. 281) und wird «von der jeweiligen Schule und Unterrichtskultur definiert» (Barth, 2012, S. 22). In diesem Sinne stellt Barth (2012) die kritische Frage, ob «die Grundschule das Recht hat, Schulfähigkeit als Eingangsbedingung vorauszusetzen oder ob sie nicht auch in der Pflicht steht, die Schulfähigkeit von Kindern zu entwickeln» (Barth, 2012, S. 23). Mit der Einteilung der Kinder mit Förderungsbedürfnis in die Einführungsklasse wird nicht nur die Exklusion gefördert, sondern auch ein Gefäss für die institutionelle Diskriminierung (Gomolla \& Radtke, 2002) geschaffen. Durch die Einführungsklassen werden soziale Differenzen hergestellt und Bildungsungleichheit reproduziert. Die Schule und auch der zur Vorschule gehörende Kindergarten erscheinen somit als Instrumente der sozialen Reproduktion. Bei der Einteilung in die Einführungsklasse sowie beim Übertritt in die zweite Klasse stehen weniger die kognitiven Fähigkeiten des Individuums, sondern Fertigkeiten wie Organisationskompetenz in Bezug auf schulische Artefakte und Instruktionsverständnis im Mittelpunkt. In diesem Sinne tritt die Selektions- und Sozialisationsaufgabe der Bildungsinstitutionen in den Vordergrund und nicht die individuelle Förderung. Steht die Einführungsklasse als Selektionsmöglichkeit im Bildungssystem zur Verfügung, greift die Schule als System auf diese Struktur zurück, um ihren Selektionsauftrag zu erfüllen und stellt damit die Grundprinzipien von social justice "the good of each and also the good of all» (Griffiths, 1998, S. 102) in Frage. Dieses Prinzip wird auch in der Entscheidung für die Wiederholung der ersten Klasse verletzt. Ob sich die Klassenwiederholung positiv auf die Lernfortschritte auswirkt, wird aufgrund empirischer Daten in Zweifel gezogen. Aus diesem Grund ist «durchaus zu verantworten, Schülerinnen und Schüler mit schwachen Schulleistungen in die nächste Klasse zu versetzen» (Bless, Schüpbach \& Bonvin, 2004, S. 139). Im Weiteren muss in Betracht gezogen werden, dass der Ausweg der Klassenwiederholung und die stillschweigende Akzeptanz von Seiten der Schule eine erhebliche Hürde für die Lernmotivation und die zukünftige Bildungskarriere des Individuums darstellen.

Hinweise zur Transkription (in Anlehnung an Langer, 2010, S. 523 und Breidenstein, 2013, S. 98)
(.) $(.).($...)
(6)
((lacht))
Pausen (1, 2, 3 Sekunden)
Pause von 6 Sekunden
Para- oder nonverbaler Akt
fett
betont gesprochen 
Da sagt der: «Komm her» gegan-

[]

$/ / / /$

$[\ldots]$
Zitat innerhalb der Rede

Wort- und Satzabbruch

Kommentare, Anmerkungen der Interviewerin

parallel gesprochen

Hinweis auf ausgelassene Textpassage

\section{Anmerkungen}

1 Die Autorin bedankt sich bei den zwei anonymen Gutachter/Innen für die wertvollen Hinweise zu diesem Beitrag.

2 Die Namen der Teilnehmenden in der Studie sind anonymisiert.

3 An dieser Stelle bedanke ich mich bei den Kolleginnen und Kollegen der Universität Zürich, insbesondere bei Frau Anna Schnitzer M.A. und bei den Teilnehmenden des Forschungskolloquiums von Frau Prof. Dr. Argyro Panagiotopoulou an der Universität zu Köln, die an der Interpretation des Datenmaterials mitgewirkt haben.

4 Quelle: http://www.avs.bl.ch/index.php?id=204 (Zugriff am 10. Januar 2013)

5 "Aufgabe: Mann zeichnen Richtzeit: 6-7 Minuten Nur mit Bleistift!

Zeichnet auf dieses leere Blatt mit dem Bleistift einen schönen grossen Mann. Ihr könnt ihn ganz so zeichnen wie ihr wollt und dürft dafür das ganze Blatt brauchen (Krebs, 1984, S. 6).»

6 Bildungsgesetz des Kantons Basel-Landschaft: http://www.baselland.ch/640-0htm.274327.0.html (Zugriff am 26. Februar 2014).

7 Ebd.

8 Ebd.

9 Die Klassenlehrperson äusserte sich im Interview auf Schweizerdeutsch. Das Interview wurde sowohl ins Schweizerdeutsche als auch ins Standarddeutsche transkribiert. Hier wird die bereinigte standarddeutsche Variante des Interviews aufgeführt.

\section{Literatur}

Annen, B., Brunner, M. \& Sollberger, J. (2005). Schulfähigkeitsbeurteilung und Schulerfolg. Psychologie und Erziehung, 2, 57-66.

Barth, K. (2012). Lernschwächen früh erkennen im Vorschul- und Grundschulalter (6. durchgesehene Aufl.). München: Ernst Reinhardt.

Baumert, J. \& Schümer, G. (2001). Familiäre Lebensverhältnisse, Bildungsbeteiligung und Kompetenzerwerb. In J. Baumert, E. Klieme, M. Neubrand, M. Prenzel, U. Schiefele, W. Schneider, P. Stanat, K.-J. Tillmann \& M. Weiss (Hrsg.), PISA 2000. Die Basiskompetenzen von Schülerinnen und Schülern im internationalen Vergleich (S. 323-407). Opladen: Leske + Budrich.

Bless, G., Schüpbach, M. \& Bonvin, P. (2004). Klassenwiederholung. Determinanten, Wirkungen und Konsequenzen. Bern: Haupt.

Bourdieu, P. \& Passeron, J.-C. (1971). Die Illusion der Chancengleichheit. Untersuchungen zur Soziologie des Bildungswesens am Beispiel Frankreichs. Stuttgart: Klett.

Breidenstein, G. (2013). Zeugnisnotenbesprechung. Zur Analyse der Praxis schulischer Leistungsbewertung. Pädagogische Fallanthologie (Band 12). Opladen: Budrich.

Charmaz, K. (2006). Constructing grounded theory. A practical guide through qualitative analysis. Los Angeles: Sage.

Christmann, N. (2009). Die Rolle der Mehrsprachigkeit im Alltag einer luxemburgischen Vorschuleinrichtung. In Ch. Röhner, C. Henrichwark, \& M. Hopf (Hrsg.), Europäisierung der Bildung. Konsequenzen und Herausforderungen für die Grundschulpädagogik (S. 108-112). Wiesbaden: VS. 
Christmann, N. (2011). Der Vielfalt (k)eine Chance geben - zur Rolle der Mehrsprachigkeit im pädagogischen Alltag einer luxemburgischen Vor- und Grundschule. In I. Diehm, \& A. Panagiotopoulou (Hrsg.), Bildungsbedingungen in europäischen Migrationsgesellschaften. Ergebnisse qualitativer Studien in Vor-und Grundschule. Sammelband der Reihe Kindheit und Kindheitsforschung (S. 73-83). Wiesbaden: VS.

Christmann, N. \& Panagiotopoulou, A. (2012). Institutionalisierte Sprachförderung für mehrsprachige Vorschulkinder: vergleichende Ethnographien in Luxemburg und Deutschland. Zeitschrift für Grundschulforschung, 5(2), 34-47.

Cuhls, A. R. \& Panagiotopoulou, A. (2010). Umgang mit Heterogenität und Förderung von Literalität am Schulanfang in einer finnischen Einheitsschule. In F. Heinzel \& A. Panagiotopoulou (Hrsg.), Qualitative Bildungsforschung im Elementar- und Primarbereich. Bedingungen und Kontexte kindlicher Lern- und Entwicklungsprozesse (S. 167-180). Hohengehren: Schneider.

Diehm, I., M. Kuhn, C. Machold \& M. Mai (2013). Ethnische Differenz und Ungleichheit. Zeitschrift für Pädagogik, 5, 644-656.

Gomolla, M. \& Radtke, F.-O. (2002). Institutionelle Diskriminierung. Die Herstellung ethnischer Differenz in der Schule. Opladen: Leske \& Budrich.

Graf. K. (2011). «Die 10 kennen wir schon vom Kindergarten.» Zur Anschlussfähigkeit von Kindergarten und Grundschule aus der Perspektive von Kindern mit Migrationshintergrund. In I. Diehm \& A. Panagiotopoulou (Hrsg.), Bildungsbedingungen in europäischen Migrationsgesellschaften. Ergebnisse qualitativer Studien in Vor- und Grundschule. Sammelband der Reihe Kindheit und Kindheitsforschung (S. 125-140). Wiesbaden: VS.

Griffiths, M. (1998). Educational research for social justice. Getting off the fence. Buckingham: Open University Press.

Hirschauer, S. \& Amman, K. (1997). Die Befremdung der eigenen Kultur. Ein Programm. In K. Amann \& S. Hirschauer (Hrsg.), Die Befremdung der eigenen Kultur. Zur ethnographischen Herausforderung soziologischer Empirie (S. 7-52). Frankfurt: Suhrkamp.

Hortsch, W. \& Panagiotopoulou, A. (Hrsg.). (2011). Sprachliche Bildung im pädagogischen Alltag. Feldstudien von angehenden GrundschullehrerInnen. Baltmannsweiler: Hohengehren.

Hortsch, W. (2010). Angebote zur Sprach(en)bildung für Kinder mit Migrationshintergrund in einer finnischen Vorschule - erste Ergebnisse einer Feldstudie. In F. Heinzel \& A. Panagiotopoulou (Hrsg.), Qualitative Bildungsforschung im Elementar-und Primarbereich. Bedingungen und Kontexte kindlicher Lern- und Entwicklungsprozesse (S. 181-191). Hohengehren: Schneider.

Hortsch, W. (2011). Kinder mit Migrationshintergrund in der finnischen Schule!? Ein Bildungssystem im Wandel? In I. Diehm, \& A. Panagiotopoulou (Hrsg.), Bildungsbedingungen in europäischen Migrationsgesellschaften. Ergebnisse qualitativer Studien in Vor-und Grundschule. Sammelband der Reihe Kindheit und Kindheitsforschung (S. 55-72). Wiesbaden: VS.

Jäger, M. (2011). «Doing difference» in einer Schweizer Primarschulklasse. Das Fremdbild des Erstklässlers Amir aus ethnographischer Perspektive. In I. Diehm \& A. Panagiotopoulou (Hrsg.), Bildungsbedingungen in europäischen Migrationsgesellschaften. Ergebnisse qualitativer Studien in Vor- und Grundschule (S. 25-44). Wiesbaden: VS.

Krebs, R. (1984). Horgener Aufgaben für das Vorschulalter: Handanweisung. Verlag: Psychologisches Institut.

Kronig, W., Haeberlin, U. \& Eckhart, M. (2000). Immigrantenkinder und schulische Selektion. Pädagogische Visionen, theoretische Erklärungen und empirische Untersuchungen zur Wirkung integrierender und separierender Schulformen in den Grundschuljahren. Bern: Haupt.

Kronig, W. (2003). Das Konstrukt des leistungsschwachen Immigrantenkindes. Zeitschrift für Erziehungswissenschaft, 6 (1), 124-139.

Kronig, W. (2007). Die systematische Zufälligkeit des Bildungserfolgs. Theoretische Erklärungen und empirische Untersuchungen zur Lernentwicklung und zur Leistungsbewertung in unterschiedlichen Schulklassen. Bern: Haupt. 
La Paro, K. M. \& Pianta, R. C. (2001). Predicting children`s competence in the early school years: A meta-analytic review. Review of Educational Research 70, 443-484.

Langer, A. (2010). Transkribieren Grundlagen und Regeln. In B. Friebertshäuser, A. Langer \& A. Prengel (Hrsg.), Handbuch Qualitative Forschungsmethoden in der Erziehungswissenschaft (3., vollständig überarbeitete Auflage), (S. 515-526). Weinheim: Juventa.

Nadai, E. \& Koch, M. (2011). Ein Forschungsobjekt im Zwischenraum. Interinstitutionelle Zusammenarbeit zwischen sozialstaatlichen Agenturen. Zeitschrift für Soziologie der Erziehung und Sozialisation (ZSE), 3, 234-247.

Maryland School Readiness Report (2011). Children entering school ready to learn. Baltimore: The Maryland State Department of Education.

Mauron Schläppi, A. (2009). Frühkindliche Bildung in Kindertagesstätten? Die Sichtweise pädagogischer Fachkräfte zum Auftrag der Kindertagestätten. Unveröffentlichte Masterarbeit, Universität Fribourg, Schweiz.

Neumann, S. (2013). Unter Beobachtung. Ethnographische Forschung im frühpädagogischen Feld. Zeitschrift für Soziologie der Erziehung und Sozialisation (33), 10-25.

Panagiotopoulou, A. \& Kassis, A. (in Druck). Frühkindliche Sprachförderung oder Forderung nach Sprachentrennung? Ergebnisse einer ethnographischen Feldstudie in der deutschsprachigen Schweiz. In T. Geier \& K. Zaborowski (Hrsg.), Migration: Auflösungen und Grenzziehungen Perspektiven einer erziehungswissenschaftlichen Migrationsforschung. Wiesbaden: VS.

Panagiotopoulou, A. \& Krompàk, E. (2014). Ritualisierte Mehrsprachigkeit und Umgang mit Schweizerdeutsch in vorschulischen Bildungseinrichtungen. Erste Ergebnisse einer ethnographischen Feldstudie in der Schweiz. In P. D. Knobloch, A. Müller \& S. Rühle (Hrsg.), Mehrsprachigkeit - Diversität - Internationalität. Hochschule als Bildungsraum. Festschrift für Cristina Allemann-Ghionda (S. 51-70). Münster: Waxmann.

Panagiotopoulou, A. (2010). Ethnographie und Bildungsqualität: Umgang mit Heterogenität und Förderung von Literalität im europäischen Vergleich. In F. Heinzel, W. Thole, P. Cloos \& S. Köngeter (Hrsg.), "Auf unsicherem Terrain» Ethnographische Forschung im Kontext des Bildungs- und Sozialwesens (S. 243-253). Wiesbaden: VS.

Panagiotopoulou, A. \& Graf, K. (2008). Umgang mit Heterogenität und Förderung von Literalität. In B. Hofmann \& R. Valtin (Hrsg.), Checkpoint literacy. Tagungsband zum 15. Europäischen Lesekongress 2007 in Berlin (S. 123-137). Berlin: Deutsche Gesellschaft für Lesen und Schreiben.

Rohlfs, C. (2011). Die Illusion der Chancengleichheit. Bildungsungleichheit vor dem Hintergrund Pierre Bourdieus Theorie der Sozialen Praxis. Wiesbaden: Springer.

Smith, D. E. (2005). Institutional ethnography. A sociology for people. Lanham: Rowman \& Littlefield.

Stamm, M. (2013). Das Konzept der Schulfähigkeit. Analysen und Reflexionen vor dem Hintergrund frühkindlicher Bildungsförderung. In M. Stamm \& D. Edelmann (Hrsg.), Handbuch frühkindliche Bildungsforschung (S. 273-284). Wiesbaden: Springer.

Stanat, P. (2003). Schulleistungen von Jugendlichen mit Migrationshintergrund: Differenzierung deskriptiver Befunde aus PISA und PISA-E. In J. Baumert, C. Artelt, E. Klieme, M. Neubrand, M. Prenzel, U. Schiefele, W. Schneider, K.-J. Tillmann \& M. Weiss (Hrsg.), PISA 2000 - Ein differenzierter Blick auf die Länder der Bundesrepublik Deutschland (S. 243-260). Opladen: Leske + Budrich.

Strauss, A. \& Corbin, J. (1996). Grounded Theory. Grundlagen qualitativer Sozialforschung. Weinheim: Beltz.

OECD (2006). Where immigrant students succeed. A comparative review of performance and engagement in PISA 2003. Paris: Author.

West, C. \& Fenstermaker, S. (1995). «Doing Difference.” Gender \& Society (9), 8-37.

Schlüsselwörter: Bildungskarriere, Reproduktion von Bildungsungleichheit, ethnographische Längsschnittstudie 


\section{La langue, une clé de réussite dans l'éducation? Reproduction d'inégalités dans les apprentissages au quotidien}

\section{Résumé}

La présente contribution s'intéresse, dans une approche ethnographique, à une période de transition dans la vie d'un enfant plurilingue, à la charnière entre le jardin d'enfant et l'école primaire. Prenant appui sur l'analyse de documents et de pratiques, des processus de sélection ainsi que l'apparition d'inégalités au niveau micro du quotidien pédagogique sont mis en évidence, en particulier lors de la transition concernée. Les conditions cadre contextuelles de politique éducative sont également thématisées.

Mots-clés: Parcours scolaire, transition scolaire, reproduction d'inégalités en éducation, étude ethnographique longitudinale

\section{La lingua, una chiave per il successo nell'educazione? La riproduzione della disuguaglianza nell'apprendimento quotidiano}

\section{Riassunto}

Con un approccio etnografico, questo contributo esplora il periodo di transizione nella vita del bambino plurilingue dalla scuola dell'infanzia alla scuola elementare. Basato sull'analisi della documentazione scientifica e delle pratiche, esso mette in evidenza - in questa fase di transizione dell'infanzia - i processi di selezione e l'apparizione della disuguaglianza al livello micro del quotidiano pedagogico. Vengono parimenti prese in esame le condizioni contestuali della politica educativa.

Parole chiave: Percorsi scolastici, riproduzione della disuguaglianza nella formazione, studi etnografici longitudinali 


\section{Language as key for success in education? A case study of reproduction of educational inequality in everyday school life}

\section{Summary}

This ethnographic study explores the period of transition from kindergarten to primary school with a sample of multilingual children. Analyses of the education policy frames, documents and practices are used to reconstruct the selection process, as well as the production process of educational inequality on the micro level of everyday school life. Contextual frame conditions of educational policy are also conceptualized.

Keywords: Educational path, school transition, reproduction of educational inequality, longitudinal ethnographic study 